\title{
Potencial de Transformación Maligna del Liquen Plano Oral: Estudio Retrospectivo
}

\author{
Potential Malignant Transformation of Oral Lichen Planus: Retrospective Study \\ Aline Tomaz; Willian Pecin Jacomacci" ${ }^{* *}$ José Henrique Santana Quinto*;
Vanessa Cristina Veltrini $^{* * *}$; Lilian Cristina Vessoni Iwaki ${ }^{* *+}$ \& Elen de Souza Tolentino***
}

TOMAZ, A.; JACOMACCI, W. P.; QUINTO, J. H. S.; VELTRINI, V. C.; IWAKI, L. C. V. \& TOLENTINO, E. S. Potencial de transformación maligna del liquen plano oral: estudio retrospectivo. Int. J. Odontostomat., 9(3):511-517, 2015.

RESUMEN: El liquen plano oral (LPO) es una enfermedad mucocutánea inflamatoria más común en las mujeres. Su etiología aún no es bien definida y esta condición puede estar relacionada, por ejemplo, a la ansiedad, estrés, diabetes, las enfermedades autoinmunes, infecciones y predisposición genética. EI LPO se manifiesta en dos formas principales: reticular y erosivo. El objetivo de esta investigación es, a través de un estudio retrospectivo, analizar el posible potencial maligno del LPO. Se analizaron los registros de los pacientes atendidos en dos proyectos de lesiones bucales de dos diferentes instituciones en el período de 1995 a 2014. Se recogieron datos como género, edad, presencia de enfermedad sistémica, presencia de lesiones en la piel, tabaquismo, duración de la lesión, localización, síntomas, tratamiento, proservación y transformación maligna. Los resultados mostraron que, de los 3488 analizados, 85 (2,4\%) tuvieron un diagnóstico confirmado de LPO, que afecta fundamentalmente a pacientes de sexo femenino $(85 \%)$ siendo el LP reticular más común. En relación a la transformación maligna del LPO, 1 paciente $(0,85 \%)$ tenía carcinoma de células escamosas 4 años después del diagnóstico inicial de LPO. Además, 3 pacientes mostraron atipia celular moderada en el diagnóstico. Los resultados refuerzan el potencial de transformación maligna del LPO. Aunque esta asociación es especulativa, la posibilidad debe ser considerada por los profesionales, que deben presentar estos pacientes a largos periodos de preservación.

PALABRAS CLAVE: liquen plano, diagnóstico, cáncer oral.

\section{INTRODUCCIÓN}

El liquen plano oral (LPO) se clasifica como una reacción inflamatoria de la mucosa oral, que afecta aproximadamente al $1-2 \%$ de la población, y tiene predilección por las mujeres de mediana edad (Gorsky et al., 2004). Quién primero describió el liquen plano (LP) fue Erasmus Wilson, un médico británico, en 1869, que creía que esta condición era causada por hongos. Pero las evidencias indican que esta enfermedad es un trastorno mucocutáneo inmunológicamente mediado (Neville et al., 2009).

La etiología del LP es un tema discutido entre los autores y no está completamente definida. Sin embargo, los estudios han mostrado una fuerte correlación entre el LP y el estado de estrés de los indivi- duos (Danielli et al., 2010; Sousa \& Rosa, 2005). La patogénesis todavía nos es bien definida. Según Sugerman et al. (2002), mecanismos específicos y no específicos pueden estar relacionados; en el mecanismo específico, los queratinocitos de la membrana basal presentan antígenos y los linfocitos $T$ citotóxicos causan la muerte de queratinocitos antígenos específicos. En el mecanismo no específico hay desgranulación de mastocitos y activación de metaloproteinasas de la matriz. Juntos, estos dos mecanismos pueden conducir a la acumulación de células T en la lámina propia, una interrupción de la membrana basal, migración de linfocitos $T$ intraepiteliales y la apoptosis de los queratinocitos (Sugerman et al.).

\footnotetext{
Estudiante del Curso de Postgrado de Odontología, Centro Universitario de Maringa (UniCesumar), Maringá, Brazil.

"* Estudiante del Curso de Postgrado de Odontología, Universidad Estadual de Maringá (UEM), Maringá, Brazil.

*** PhD, Profesora del Curso de Odontología del Centro Universitario de Maringá (UniCesumar) y de la Universidad Estadual de Maringá (UEM), Maringá, Brazil.
} 
EI LP puede presentarse clínicamente en seis formas: reticular, erosiva, bullosa, placa, papular y atrófica (Mollaoglu, 2000), siendo los dos primeros las formas principales. El LP reticular aparece con más frecuencia, afecta la región posterior mucosa bucal bilateralmente y puede ocurrir en otras regiones, como el borde lateral y dorso de la lengua, las encías, el paladar y los labios. Se manifiesta como estrías blancas entrelazadas, más comúnmente asintomáticas. El LP erosivo no es tan común, pero es más importante, teniendo en cuenta que estas lesiones tienen síntomas y es clínicamente posible visualizar áreas eritematosas atróficas con diversos grados de ulceración central. En los extremos de las regiones atróficas se observan finas rayas blancas irradiadas (Neville et al.).

Histopatológicamente se observa una licuefacción de la capa basal, intensa infiltración de linfocitos subepiteliales, degeneración de queratinocitos en ceratinóticos en la región epitelial y en el tejido conectivo (cuerpos de Civatte), en la espesura de la capa espinosa y una variación en el grado de ortoqueratosis y paraqueratosis (Edwards \& Kelsh, 2002; Neville et al.; Eisenberg, 2000; Regezi \& Sciubba, 2000).

El diagnóstico del LP se hace generalmente por medio de exámenes clínicos e histológicos. Sin embargo, el diagnóstico de la forma reticular a menudo se basa sólo en el examen clínico (Rosa, 2011). Con ninguna cura, fármacos anti-inflamatorios esteroideos se pueden utilizar para controlar los síntomas, cuando existan. McCartan \& Healy (2008) y Kesic et al. (2009) reportan que los irritantes también deben ser identificados y eliminados, como el alcohol, el tabaco, dientes fracturados y prótesis dentales mal ajustadas.

Como no existe un tratamiento específico para el LP, existe la necesidad de controlar a los pacientes durante un período prolongado, con el fin de que algunos casos pueden sufrir transformación maligna (Fang et al., 2009). Quien primero describió un caso de desarrollo de LPO para el carcinoma de células escamosas fue Hallopeau en 1910 (Rosa, 2011). Desde entonces se han realizado estudios para analizar el posible potencial maligno del LPO, y muchos estudios demostraron ese potencial. Diferentes trabajos muestran una frecuencia de transformación de 0,4\% a 5,3\% (Shi et al., 2010). Por esta razón, la Organización Mundial de la Salud (OMS) ha clasificado el LPO como un trastorno potencialmente maligno (El Naggar, Reichart, 2005).
Sin embargo, la verdadera razón para esa transformación no es conocida (Sousa \& Rosa, 2008). Según Mignona et al. (2004), procesos inflamatorios crónicos, tales como el LPO, generarían un microambiente basado en citoquinas que tienen la capacidad de influir en la supervivencia celular, alterando su crecimiento y diferenciación, para favorecer la iniciación, promoción y progresión del cáncer.

Mientras que el potencial maligno del LPO es bastante controversial, muchos profesionales no lo consideran un trastorno potencialmente maligno, y por lo tanto no siguen los pacientes durante un largo período, el objetivo de este estudio es realizar un estudio observacional retrospectivo para analizar el posible potencial transformación maligna del LPO, así como relatar un caso clínico relacionado con el tema.

\section{MATERIAL Y MÉTODO}

A partir de un estudio observacional y retrospectivo, fueron analizados los registros médicos de la época de 2004 a 2010 del Proyecto PROLEB de la Unicesumar, y del período de 1995 a 2014 del Proyecto LEBU de la Universidad Estadual de Maringá (UEM), teniendo en cuenta que este fue el período de validez de los dos proyectos en las instituciones, con el fin de seleccionar todos los casos diagnosticados clínica e histopatológicamente como LPO.

En estos registros seleccionados, se obtuvieron los datos, teniendo en cuenta las variables: género, edad, origen étnico, presencia de enfermedad sistémica, presencia de lesiones en la piel, asociación con Candida albicans, tabaquismo, duración de la lesión, localización, síntomas, antecedentes familiares, tratamiento, proservación y transformación maligna.

La transformación maligna fue identificada a través de pruebas en el examen histopatológico realizado durante el seguimiento de los pacientes.

Una base de datos de las variables fue organizada para permitir la tabulación y análisis estadístico utilizando la prueba no paramétrica de chi-cuadrado $(p<0,05)$.

Este estudio fue aprobado por el Comité de Ética en la investigación con seres humanos, Protocolo 344.003. 


\section{RESULTADOS}

Se analizaron 2.702 registros del Proyecto LEBU y 786 registros del PROLEB, totalizando 3.488 registros. De ellos, 85 (2,4\%) eran de LP. En todos los casos, se realizó una biopsia.

Cuánto al tipo de LP, 65 pacientes presentaron el tipo reticular $(76,5 \%), 12(14,1 \%)$ el tipo erosivo, 6 pacientes $(7 \%)$ con asociación reticular/erosivo, 1 $(1,2 \%)$ con el tipo papular, y $1(1,2 \%)$ tipo bulosa (Fig. 1). Hubo una diferencia estadísticamente significativa $(p<0,001)$ entre el LP reticular y todos los demás tipos.

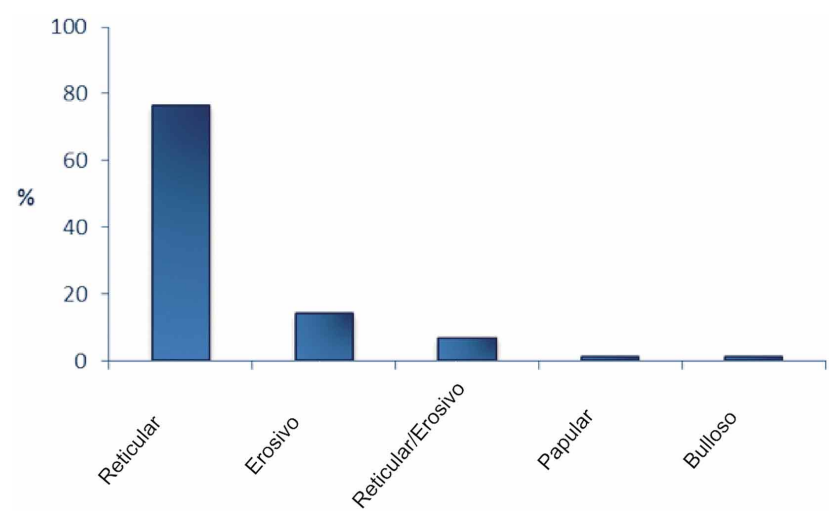

Fig. 1. Distribución de los pacientes según el tipo de liquen plano $(p<0,001)$.

De los 85 pacientes, 17 eran fumadores $(20 \%)$. 41 pacientes $(48,2 \%)$ presentaron algún tipo de dolor quemante o síntomas. Sólo 4 pacientes $(4,7 \%)$ también tenían lesiones en la piel. En relación con el sexo, 68 eran mujeres (80\%), y 17 eran varones (20\%) (Fig. 2), con diferencias estadísticamente significativas $(p<0,05)$.

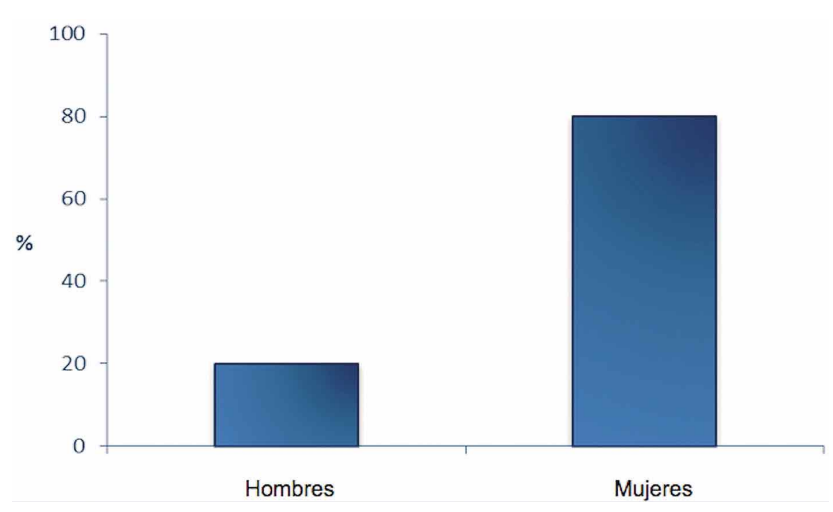

Fig. 2. Distribución de los pacientes según el sexo $(p<0,05)$.
En referencia a la edad, 6 pacientes tenían entre 20 a 30 años (7\%), $19(22,4 \%)$ entre $31-40$ años $19(22,4 \%), 28(32,9 \%)$ entre $41-50$ años, 17 entre 51-60 años (20\%), 13 pacientes eran mayores de 60 años $(15,3 \%)$ y 2 pacientes no informaron la edad $(2,4 \%)$ (Fig. 3). No hubo diferencia estadísticamente significativa $(p>0,05)$.

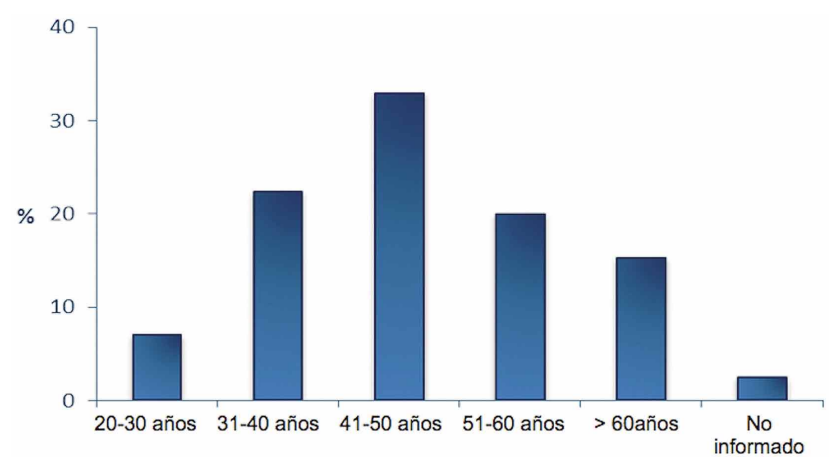

Fig. 3. Distribución de los pacientes según la edad ( $p>0,05)$.

El tratamiento se produjo en 34 pacientes sólo a través de la observación (40\%), en 23 pacientes la administración de la medicación local y sistémica $(27,1 \%), 7$ pacientes fueron tratados con medicación sistémica y local y se remitieron también a los dermatólogos (8.2\%), 16 pacientes fueron remitidos directamente a un médico/dermatólogo (18,8\%), en 1 paciente se realizó la extirpación quirúrgica $(1,2 \%)$, y en 1 se cambió la restauración de amalgama $(1,2 \%)$. En los registros 3 pacientes no se presentaba el tratamiento $(3,5 \%)$.

Con respecto a la transformación maligna del LP, de 85 pacientes, 4 (4,7\%) mostraron atipia celular: $3(3,5 \%)$ con atipia moderada y uno con carcinoma de células escamosas (0,85\%) (Fig. 4). El paciente que desarrolló carcinoma de células escamosas era mujer, presentaba el tipo reticular de LP y su edad era de 60 años. De los 3 pacientes con atipia moderada, 2 fueron $(66,6 \%)$ eran mujeres y un hombre $(33,3 \%)$. Cuánto al tipo de LP, todos tenían el tipo erosivo (100\%). Cuánto a la edad de estos pacientes, 1 tenía entre 31 a 40 años (33,3\%), uno entre 51 a 60 años $(33,3 \%)$ y 1 paciente tenía más de 60 años $(33,3 \%)$. No hubo diferencia estadísticamente significativa ( $p>$ 0,05 ). Las atipias celulares moderadas fueron encontradas en las biopsias realizadas para el diagnóstico del LPO. Apenas el caso de carcinoma de células escamosas fue diagnosticado en la biopsia 4 años después del diagnóstico inicial de LP. 
TOMAZ, A.; JACOMACCI, W. P.; QUINTO, J. H. S.; VELTRINI, V. C.; IWAKI, L. C. V. \& TOLENTINO, E. S. Potencial de transformación maligna del liquen plano oral: estudio retrospectivo. Int. J. Odontostomat., 9(3):511-517, 2015.

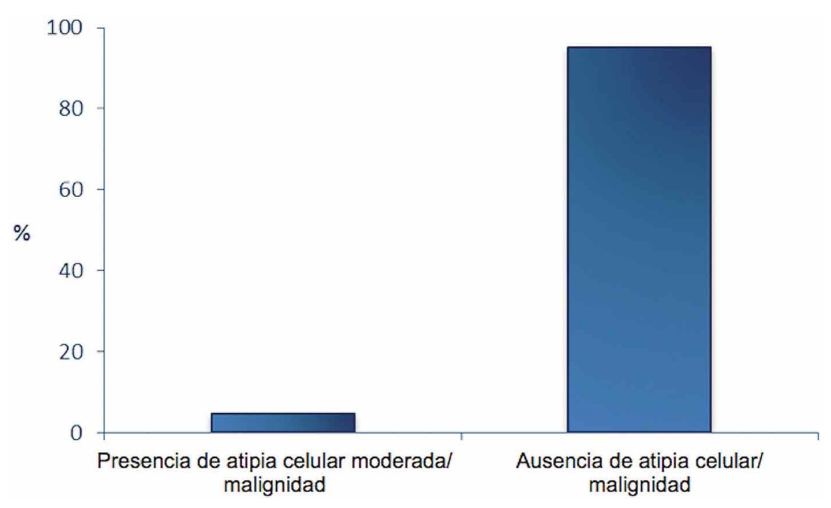

Fig. 4. Distribución de la atipia moderada y transformación maligna, cuanto al número total de pacientes con liquen plano $(p>0,05)$.
En un caso de una paciente de sexo femenino, de 74 años, leucoderma, asistió a la Clínica Dental de la Universidad Estadual de Maringá (UEM) en 2012, quejándose de un gran dolor en las encías. En la exploración física se observó una placa blanca con aspecto irregular en la encía adherida entre canino maxilar y el primer molar maxilar en el lado izquierdo (Fig. $5 A$ ). Radiográficamente fue detectado la presencia del implante en la región el primero premolar y el hueso con la aparición de normalidad. Durante la entrevista, el paciente informó que hace dos años se había sometido a una biopsia en la misma región y que el diagnóstico fue de LP reticular. La decisión en ese momento fue la aplicación tópica de acetónido de

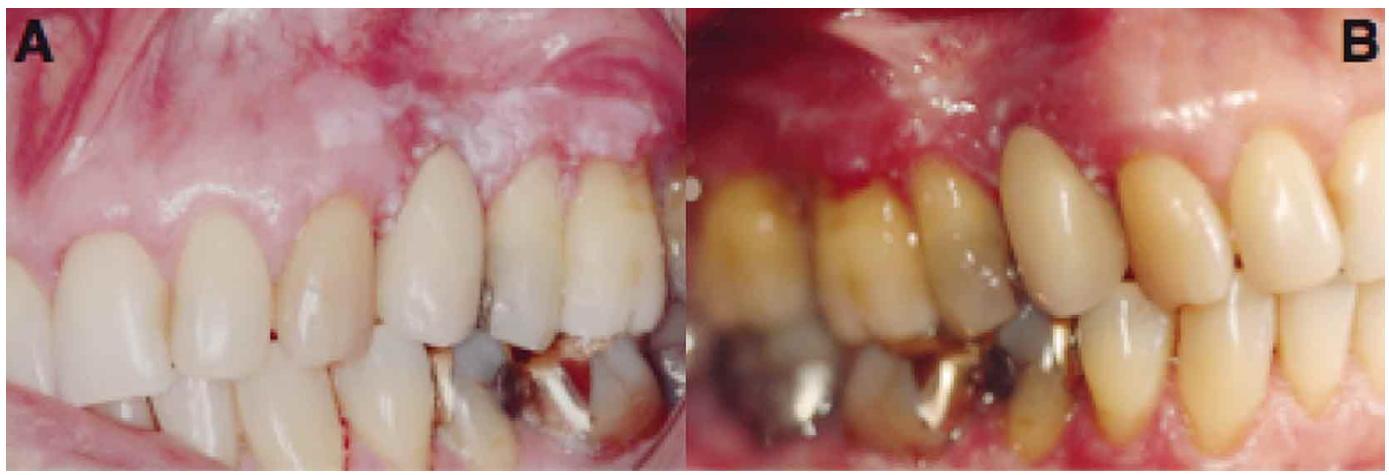

Fig. 5. A) Aspecto clínico inicial de las lesiones orales. Placa blanca en la región de la encía bucal entre el diente canino y el primer molar maxilar en el lado izquierdo. B) Mejora clínica de las lesiones después de 14 días de tratamiento.

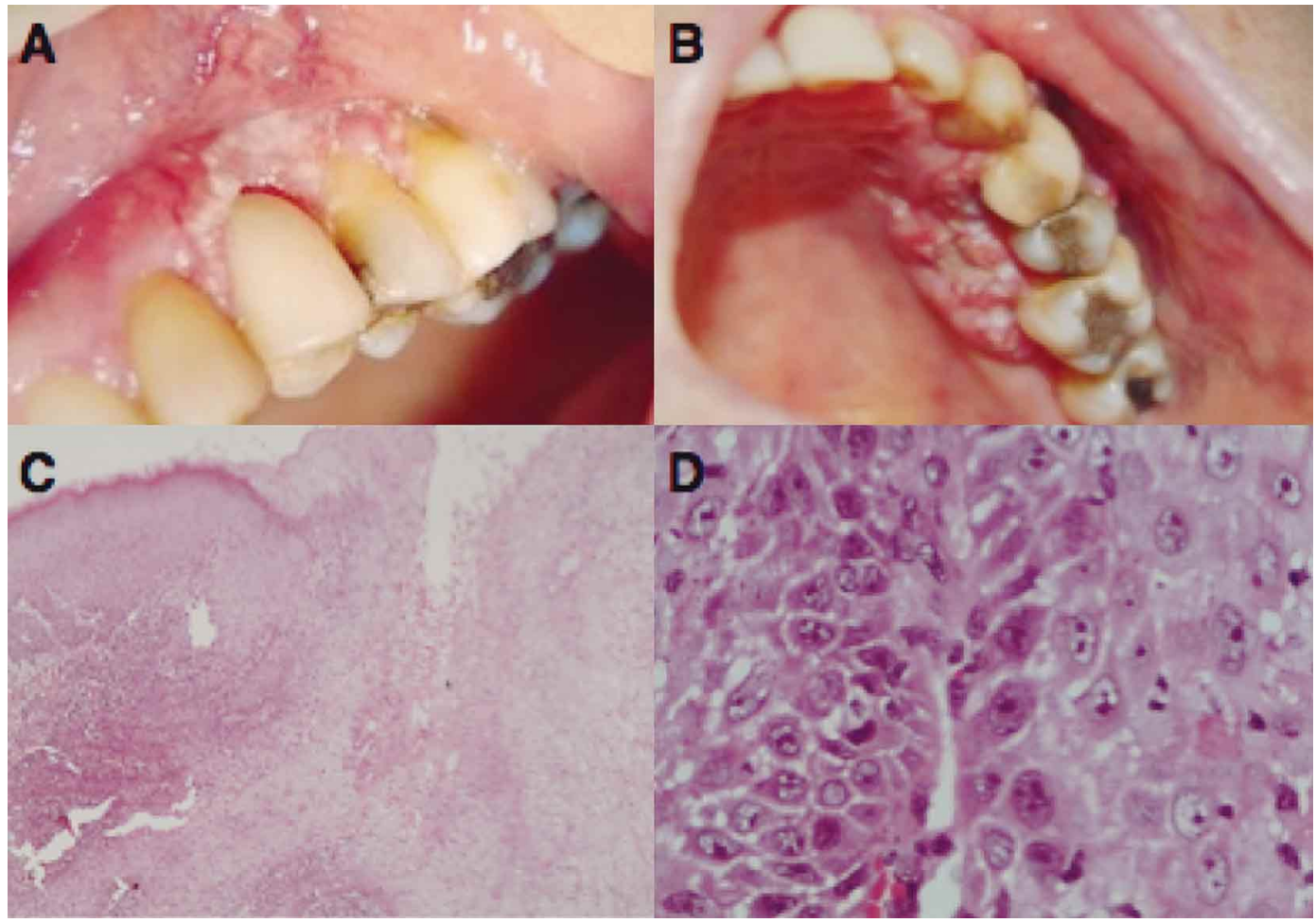

Fig. 6. Aspecto clínico y fotomicrografías de las biopsias de la lesión en el retorno. A) placa eritroleucoplásica en la región de la encía entre el canino superior y el primer molar en el lado izquierdo. B) Lesión con aspecto irregular en el paladar del lado izquierdo, entre el canino y el primer molar. C) patrón romboidal de invasión (aumento de 4x) D) células neoplásicas relativamente pleomórficas y hipercromáticas (aumento de x 40). 
triamcinolona y dipropionato de beclometasona con el fin de aliviar los síntomas mediante la modulación de la respuesta inflamatoria y inmune. Después de 14 días de uso de los corticosteroides tópicos, el paciente mejoró clínicamente y de la sintomatología (Fig. 5B).

Dos años más tarde (4 años después del diagnóstico inicial de LP) la paciente volvió a quejarse de nuevos síntomas de dolor. A continuación, la lesión se presentaba con empeoramiento del aspecto clínico, con superficie verrugosa de la encía (Fig. 6A) y de la mucosa palatina (Fig. 6B). Nuevas biopsias incisionales se realizaron en encía bucal y mucosa palatina. El examen histopatológico reveló un carcinoma escamoso invasivo, pero bien diferenciado (Fig. $6 \mathrm{C}$ y D). El paciente fue remitido para tratamiento quirúrgico por el equipo oncológico.

\section{DISCUSIÓN}

En el presente estudio, se observó que la mayoría de pacientes con LP es de sexo femenino (80\%), apoyando la gran mayoría de los estudios, como Markopoulos et al. (1997) y Shen et al. (2012). Se sabe que la variante reticular es la más frecuente Bardellini et al. (2013), lo que se confirmó en este estudio (75,3\% de los casos). Sólo 4 pacientes (4,7\%) tenían lesiones concomitantes en piel, así como Bidarra et al. (2008), que también encontraron pacientes con lesiones de LPO asociadas con lesiones cutáneas.

En cuanto al potencial maligno del LP, 1 paciente presentó un carcinoma de células escamosas, o sea, la propia transformación maligna, lo que correspondió a $0,85 \%$ de los casos. Estos resultados son similares a los presentados por Rajentheran et al. (1999), donde de 832 casos diagnosticados con LP, $0,8 \%$ sufrieron transformación. Sin embargo los datos difieren de los del estudio de Tizeira et al. (2003), donde se produjo la transformación maligna en un mayor porcentaje de casos $(6,51 \%)$. Si los casos deatipia moderada fueran registrados en el presente estudio, serían 4,7\% de los pacientes afectados. Respecto al tipo de LP, los estudios corroboran entre sí, y el LP erosivo es más propenso a someterse a transformación maligna. Sin embargo, en este estudio el caso de la transformación fue diagnosticado previamente como LP reticular.

Hay estudios, como el de Sousa et al. (2005), quienes concuerdan con esto y muchos otros estu- dios que muestran la capacidad de transformación maligna del LP. Los autores no encontraron ninguna relación entre el LP y el cáncer, y muestran las diferencias de perfil entre los pacientes con carcinoma y con LP, y todavía dicen que los casos de transformación maligna reportados en la literatura pueden estar relacionados con errores en el diagnóstico histopatológico. Un estudio realizado por Danielli et al. evaluaron 52 casos confirmados de LP y ningún caso presentó características clínicas o histopatológicas de transformación maligna. Sin embargo, para los autores, la posibilidad mínima de esa transformación ocurrir hace necesaria la aplicación de normas estrictas y un control sistemático de estos pacientes durante largos períodos. Shen et al. afirman que algunas lesiones diagnosticadas como LP tratan de lesiones displásicas con aspecto liquenoide, y que datos no obtenidos en relación con el tabaco pueden llevar a una alteración maligna superpuesta y esto no es una evolución de la anterior. Para Shen et al. y Bardellini et al., el tabaco no es un agente que influye en el proceso de transformación maligna del LP, una vez que en los casos donde hubo una transformación maligna, los pacientes negaron ser fumadores. Los resultados obtenidos en este estudio están de acuerdo con estos autores, ya que el único caso de neoplasia, la paciente no fumaba. Sin embargo, se observó que en 3 casos con atipia celular moderada los pacientes reportaron ser fumadores, un hecho que debe ser tomado en consideración.

En cuanto a la edad de los pacientes cuando se produjo la transformación maligna, los presentes resultados corroboran el trabajo de Fang et al., quienes reportaron la ocurrencia de transformación en los pacientes de más edad. La paciente en este estudio tenía más de 60 años.

Cómo se produce la transformación maligna LP también es discutible entre los autores. Mignona et al. creen que los procesos inflamatorios crónicos tales como el LP crean un microambiente que se basa en las citoquinas que influyen en última instancia, sobre la supervivencia de las células y en consecuencia en el crecimiento y diferenciación, promoviendo así la iniciación, promoción y progresión del cáncer. Ya Bascones et al. (2005) analizaron la influencia de la detención del ciclo celular y la apoptosis en el proceso de transformación maligna del LP, a través de la evaluación del índice de apoptosis mediante el método TUNEL (Terminal transferasa mediada dUTP deoxyribonucleotidyl Nick 
End Labeling) y la expresión inmunohistoquímica de las proteínas Bax, caspasa-3 y p21, llegaron a la conclusión de que la transformación puede producirse por la baja respuesta de las células epiteliales a los mecanismos de detención del ciclo celular y de la apoptosis.

En cuanto al tiempo medio entre el diagnóstico del LP y su transformación maligna, la paciente en este estudio tenía un diagnóstico de carcinoma de células escamosas 4 años después del diagnóstico inicial de LP. En la literatura, el tiempo promedio de trasformación fue mayor. Markopoulos et al. reportaron un promedio de 6,5 años después del diagnóstico inicial; ya Rajentheran et al. encontraron un promedio de 9 años. En un estudio más reciente, Shen et al. encontraron un promedio de 5,8 años.
Estos datos refuerzan la importancia de la proservación del LPO por períodos prolongados.

\section{CONCLUSIÓN}

Los resultados de este estudio refuerzan el potencial de transformación maligna del LP. Aunque la asociación de la enfermedad con el cáncer es especulativa, esta posibilidad debe ser considerada por los profesionales, que deben presentar estos pacientes a largos periodos de monitoreo. En cualquier signos clínicos sospechosos se debe realizar una nueva biopsia. El hábito de fumar, la variante erosiva de la enfermedad y la vejez parecen jugar un papel importante en la transformación maligna de LP oral.

TOMAZ, A.; JACOMACCI, W. P.; QUINTO, J. H. S.; VELTRINI, V. C.; IWAKI, L. C. V. \& TOLENTINO, E. S. Potential malignant transformation of oral lichen planus: Retrospective study. Int. J. Odontostomat., 9(3):511-517, 2015.

ABSTRACT: Oral lichen planus (OLP) is an inflammatory mucocutaneous disease, more common in females. Its etiology is not yet well defined; this condition may be related, for example, to anxiety, stress, diabetes, autoimmune diseases, infections and genetic predisposition. The OLP manifests itself in two main forms: reticular and erosive. This work aims to perform a retrospective study analyzing the possible malignant potential of OLP. Records of the patients were analyzed in two projects of oral lesions in two different institutions in the period from 1995 to 2014. Data as gender, age, presence of systemic disease, presence of skin lesions, smoking, duration of the injury, location, symptoms, treatment, follow-up time and malignant transformation were collected. The results showed that, of the 3488 analyzed, $85(2.4 \%)$ had a confirmed diagnosis of OLP, which mainly affected female patients (85\%) being the most common the reticular LP. Regarding the malignant transformation of OLP, one patient $(0.85 \%)$ had squamous cell carcinoma 4 years after the initial diagnosis of OLP. In addition, 3 patients showed moderate cellular atypia. The results reinforce the potential of malignant transformation of OLP. Although this association is speculative, this possibility must be considered by the dental practitioners, who must submit these patients to long periods of monitoring.

KEY WORDS: lichen planus, diagnosis, oral cancer.

\section{REFERENCIAS BIBLIOGRÁFICAS}

Bardellini, E.; Amadori, F.; Flocchini, P.; Bonadeo, S. \& Majorana, A. Clinicopathological features and malignant transformation of oral lichen planus: a 12-years retrospective study. Acta Odontol. Scand., 71(3-4):83440, 2013.

Bascones, C.; Gonzales-Moles, M. A.; Esparza, G.; Bravo, M.; Acevedo, A.; Gil-Montoya, J. A. \& Bascones, A. Apoptosis and cell cycle arrest in oral lichen planus Hypothesis on their possible influence on its malignant transformation. Arch. Oral Biol., 50(10):873-81, 2005.

Bidarra, M.; Buchanan J. G.; Scully C.; Moles, D. R. \& Porter, S. R. Oral lichen planus: a condition with more persistence and extra-oral involvement than suspected? Oral Pathol. Med., 37(10):582-6, 2008.
Danielli, J.; Vilanova, L. S. R.; Pedroso, L. \& Silva, M. A. G. Protocolo de atendimento e acompanhamento do paciente com líquen plano oral (LPO). Rev. Odontol. Bras. Central, 19(50):233-8, 2010.

Edwards, P. C. \& Kelsh, R. Oral lichen planus: clinical presentation and management. J. Can. Dent. Assoc., 68(8):494-9, 2002.

Eisenberg, E. Oral lichen planus: a benign lesion. J. Oral Maxillofac. Surg., 58(8):1278-85, 2000.

El Naggar, A. K. \& Reichart, P. A. Proliferative verrucous leukoplakia and precancerous condition. In: Barnes, L.; Eveson, J. W.; Reichart, P. \& Sidransky, D. World Health Organization Classification of Tumours. Lyon, IARC Press, 2005. 
Fang, M.; Zhang, W.; Chen, Y. \& He, Z. Malignant transformation of oral lichen planus: a retrospective study of 23 cases. Quintessence Int., 40(3):235-42, 2009.

Gorsky, M.; Epstein, J. B.; Hasson-Kanfi, H. \& Kaufman, E. Smoking habits among patients diagnosed with oral lichen planus. Tob. Induc. Dis., 2(2):103-8, 2004.

Kesic, L.; Obradovic, R.; Mihailovic, D.; Radicevic, G.; Stankovic, S. \& Todorovic, K. Incidence and treatment outcome of oral lichen planus in southeast Serbia in a 10-year period (1997-2007). Vojnosanit. Pregl., 66(6):435-9, 2009.

Markopoulos, A. K.; Antoniades, D.; Papanayotou, P. \& Trigonidis, G. Malignant potential of oral lichen planus; a follow-up study of 326 patients. Oral Oncol., 33(4):2639, 1997.

McCartan, B. E. \& Healy, C. M. The reported prevalence of oral lichen planus: a review and critique. J. Oral Pathol. Med., 37(8):447-53, 2008.

Mignogna, M. D.; Fedele, S.; Lo Russo, L.; Lo Muzio, L. \& Bucci, E. Immune activation and chronic inflammation as the cause of malignancy in oral lichen planus: is there any evidence? Oral Oncol., 40(2):120-30, 2004.

Mollaoglu, N. Oral lichen planus: a review. Br. J. Oral Maxillofac. Surg., 38(4):370-7, 2000.

Neville, B. W.; Damm, D. D.; Allen, C, M. \& Bouquot, J. E. Doenças dermatológicas. In: Neville, B. W.; Damm, D. D.; Allen, C. M.; Bouquot, J. E. Patologia epitelial. 3rd ed. Rio de Janeiro, Elsevier. 2009. pp.743-817.

Rajentheran, R.; McLean, N. R.; Kelly, C. G.; Reed, M. F. \& Nolan, A. Malignant transformation of oral lichen planus. Eur. J. Surg. Oncol., 25(5):520-3, 1999.

Regezi, J. A. \& Sciubba, J. J. Patologia bucal: correlações clínicopatológicas. $3 r^{d}$ ed. Rio de Janeiro, Guanabara Koogan, 2000.

Rosa, R. R. Reavaliação dos pacientes com líquen plano oral atendidos no período de 1997 a 2010. Dissertação. Uberlândia, Universidade Federal de Uberlândia, 2011.

Shen, Z. Y.; Liu, W.; Zhu, L. K.; Feng, J. Q.; Tang, G. Y. \& Zhou, Z. T. A retrospective clinicopathological study on oral lichen planus and malignant transformation: analysis of 518 cases. Med. Oral Patol. Oral Cir. Bucal, 17(6):e943-7, 2012.

Shi, P.; Liu, W.; Zhou, Z. T.; He, Q. B. \& Jiang, W. W. Podoplanin and ABCG2: malignant transformation risk markers for oral lichen planus. Cancer Epidemiol. Biomarkes Prev., 19(3):844-9, 2010.
Sousa, F. A. C. G.; Fontes, P. C.; Bolanho, A. \& Rosa, L. E. $B$. Estudo comparativo entre o líquen plano e o carcinoma epidermóide em mucosa bucal. Cienc. Odontol. Bras., 8(1):55-60, 2005.

Sousa, F. A. C. G. \& Rosa, L. E. B. Líquen plano bucal: considerações clínicas e histopatológicas. Rev. Bras. Otorrinolaringol., 74(2):284-92, 2008.

Sousa, F. A. C. G. \& Rosa, L. E. B. Perfil epidemiológico dos casos de líquen plano oral pertencentes aos arquivos da disciplina de patologia bucal da Faculdade de Odontologia de São José dos Campos - UNESP. Cienc. Odontol. Bras., 8(4):96-100, 2005.

Sugerman, P. B; Savage, N. W.; Walsh, L. J.; Zhao, Z. Z.; Zhou, X. J.; Khan, A.; Seymour, G. J. \& Bigby, M. The pathogenesis of oral lichen planus. Crit. Ver. Oral Biol. Med., 13(4):350-65, 2002.

Tizeira, H. E. L.; Aguas, S. C. \& Sano, S. M. Transformación maligna del liquen plano bucal atípico: Análisis de 32 casos. Med. Oral, 8:2-9, 2003.

Dirección para Correspondencia:

Willian Pecin Jacomacci

Universidade Estadual de Maringá

Departamento de Odontologia

Av. Mandacaru, 1550. CEP 87080-000

Maringá - PR

BRASIL

Email: willianpjacomacci@gmail.com

Recibido : 20-10-2014

Aceptado: 17-11-2015 